\title{
Inter-specific variability in protein use by two vegetable crop species
}

\section{Bartosz Adamczyk*, Mirosław Godlewski}

Department of Plant Cytology and Cytochemistry, Institute of Plant Physiology, Cytology and Cytogenetic, University of Łódź, 90-237 Łódź, Poland

*Corresponding author: Bartosz Adamczyk, Department of Plant Cytology and Cytochemistry, Institute of Plant
Physiology, Cytology and Cytogenetic, University of Łódź, Poland, e-mail: bartek adamczyk 79@02.pl

Received: 04 May 2010; Accepted: 27 September 2010

\begin{abstract}
It is now well-known that plants can uptake not only inorganic nitrogen but also organic nitrogen compounds, mainly amino acids. However, soil proteins are the main pool of amino acids. According to our earlier papers, plants can get access to this source of nitrogen using root-secreted proteases, but the level of proteolytic activity of such root-secreted proteases is species-specific. Our aim was to compare the use of protein as nitrogen source by two vegetable crops having high (Allium porrum) or low (Lactuca sativa) level of activity of root-secreted proteases. Seedlings were cultivated on Murashige and Skoog medium (MS), MS medium without inorganic nitrogen, MS medium without inorganic nitrogen, but with casein in concentration of $0.01 \%, 0.1 \%$ or $1 \%$. Fresh weight of shoot of $A$. porrum was the highest for seedlings growing on culture medium with casein, but shoots of $L$. sativa obtained the highest weight growing on the culture medium with inorganic nitrogen. Allium porrum seedlings obtained 15 -fold higher proteolytic activity in the culture medium than $L$. sativa. Seedlings of $A$. porrum using such high activity of proteases secreted by roots could provide a substantial pool of amino acids for intensive growth. The current studies conducted on $A$. porrum and $L$. sativa suggest that the efficiency of protein use in nitrogen nutrition by plants is species-specific.
\end{abstract}

Keywords: organic nitrogen, plant nitrogen nutrition, secretion of proteases

\section{INTRODUCTION}

It is now well established that plants can uptake not only inorganic nitrogen, but also organic forms of this nutrient, mainly amino acids, even in the presence of microbial competitors (Lipson and Näsholm, 2001; Ge et al., 2009; Näsholm et al., 2009), and especially in conditions of high amino acid concentrations in the soil (Jones et al., 2005; Sauheitl et al., 2009). The ability of plants to uptake amino acids was proven in numerous experiments for many plant species (i.e. Falkengren-Grerup et al., 2000; Persson and Näsholm, 2001; Xu et al., 2004; Hirner et al., 2006; Rentsch et al., 2007; Svennerstam et al., 2007). Amino acids are the major fraction of soil nitrogen; however, they are mainly present in polymeric form as proteins (Kaye and Hart, 1997). Protein utilization therefore is associated with proteolysis driven by microbial proteases. Our earlier studies showed, that also plants can secrete proteases by intact roots (Godlewski and Adamczyk, 2007) and that Triticum aestivum roots can directly utilize proteins in the culture medium as a source of nitrogen without prior digestion by microbial proteases (Adamczyk et al., 2008). This phenomenon was also proven by Paungfoo-Lonhienne et al., (2008); their studies were conducted on two non-mycorrhizal species - Arabidopsis thaliana and Hakea actities; additionally these authors showed that Arabidopsis thaliana growth was supported better by protein and low inorganic nitrogen than protein or low inorganic nitrogen alone. Moreover, Paungfoo-Lonhienne 
et al. (2008) suggested a possible uptake of intact protein by plant roots via endocytosis.

In our earlier paper we proved that proteases secreted by plant roots can digest proteins (casein, bovine serum albumin) to low-molecular-mass products (Adamczyk et al., 2009) which can be taken up directly by plant roots (Rentsch et al., 2007; Tsay et al., 2007; Paungfoo-Lonhienne et al., 2009). However, the level of proteolytic activity in the culture medium of different plant species varied significantly (Godlewski and Adamczyk, 2007). Also the uptake of amino acids - the product of proteolysis process - differs between various plant species (Weigelt et al., 2003, 2005; Okamoto and Okada, 2004; Reeve et al., 2009).

In this paper we were looking for a linkage between these differences in organic nitrogen uptake and the level of proteolytic activity in the culture medium. We were studying if there are some differences in the use of proteins by various plant species - Allium porrum cv. Bartek and Lactuca sativa $\mathrm{cv}$. Ewelina. We have chosen these species because of high discrepancy between their root-secreted proteolytic activities in the culture medium in preliminary studies $-A$. porrum had the highest and $L$. sativa had the lowest proteolytic activity, comparing to other studied species (Godlewski and Adamczyk, 2007). These plant species were cultivated in sterile conditions on Murashige and Skoog medium (MS; Murashige and Skoog, 1962), MS medium without inorganic nitrogen, MS medium without inorganic nitrogen, but with casein $(0.01 \%, 0.1 \%$ or $1 \%)$.

Our hypotheses were that (1) A. porrum seedlings would have higher shoot weight relative to $L$. sativa, growing on medium containing protein (2) $L$. sativa would be less effective at growing on protein compared to $A$. porrum seedlings (3) these variations in protein use could be reflected also in the level of proteolytic activities in the culture medium.

\section{MATERIALS AND METHODS}

Plant material and cultivation: Seeds of Allium porrum L. cv. Bartek and Lactuca sativa cv. Ewelina were obtained from a commercial plant distributor (Torseed, Poland). Plants were cultivated in aseptic conditions as in our previous studies (Godlewski and Adamczyk, 2007). Briefly, seeds were surface sterilized with $70 \%$ ethyl alcohol and with $10 \%$ sodium hypochlorite; and germinated on Petri dishes (7 days) and later separately in tubes (for 2 weeks; whole experiment took 3 weeks), containing $15 \mathrm{ml}$ of autoclaved liquid medium: Murashige and Skoog medium (MS), MS medium without inorganic nitrogen, MS medium without inorganic nitrogen, but with casein in concentration of $0.01 \%, 0.1 \%$ or $1 \%$. MS medium without modifications includes $60 \mathrm{mM}$ of inorganic nitrogen, MS medium without inorganic nitrogen but with casein included: $1.18 \mathrm{mM}, 11.8 \mathrm{mM}$ and $118 \mathrm{mM}$ of nitrogen for $0.01 \%, 0.1 \%$ and $1 \%$ casein concentration, respectively. The experiment was performed on liquid medium instead of sterilized soil, because soil contains both, inorganic and organic sources of nitrogen, so it would not be possible to obtain very controlled conditions of nitrogen sources. The seedlings were cultivated at $23 \pm 1{ }^{\circ} \mathrm{C}$ air temperature, $70 \%$ relative humidity, and 16:8 h photoperiod with $380 \mu \mathrm{mol}$ m-2 s-1 light intensity at plant height. Sterility in the culture medium after cultivation and purification was verified with microbiological tests (Microcount combi; Schülke-Mayr).

Proteolytic activity measurements: The culture medium was purified and concentrated using a Vivapore 5 solvent absorption concentrator (Sigma) with pore size 7500 MWCO. Proteolytic activity was determined using method by Tomarelli et al. (1949), in which the time-dependent release of azo dyecoupled trichloroacetic acid soluble peptide fragments from the substrate, azocasein, was monitored. Briefly, $200 \mu \mathrm{l}$ of partially purified and concentrated culture medium was mixed with $100 \mu \mathrm{l} 0.5 \%(\mathrm{w} / \mathrm{v})$ azocasein dissolved in $0.9 \% \mathrm{NaCl}$ in $50 \mathrm{mM}$ phosphate buffer (pH 6.8). After $4 \mathrm{~h}$ incubation, the reaction was stopped by adding $200 \mu \mathrm{l} 20 \%$ (w/v) trichloroacetic acid. After centrifugation (10000g, $5 \mathrm{~min}$.), $150 \mu \mathrm{l}$ of the resulting supernatant was mixed with $50 \mu \mathrm{l} 1 \mathrm{M}$ $\mathrm{NaOH}$, and after 30 minutes absorbance at $440 \mathrm{~nm}$ was read (Hitachi U-2000 Spectrophotometer, Japan). In the control, trichloroacetic acid was added to the culture medium prior to addition of azocasein. One unit of protease activity was defined as the amount of enzyme that increased the absorbance by 0.1 at $440 \mathrm{~nm}$ per $1 \mathrm{~h}$. All reagents were purchased from Sigma. Proteolytic activities are presented per one square millimeter of the root surface, which was measured with stereological method, as described by Head (1966), on the basis of length of the root and root fresh weight.

Azocasein method was chosen for this study because it is a nonspecific protease substrate, what means that azocasein 
can be easily digested by different proteases. Azocasein is often used for measuring the overall proteolytic activity (Hano et al., 2008). Methods of measurements of proteolytic activity on the basis of azocasein digestion are widely used (i.e. Zhang et al., 2007; Rojas et al., 2009).

Statistics: Each experiment consisted of 6 replicates $(n=6)$. Proteolytic activity means were compared among nitrogen treatments and fresh weight means were compared among nitrogen treatments using one-way ANOVA, followed by Tukey's test. We used Statistica (Statsoft, Inc.).

\section{RESULTS}

Fresh weight of roots and shoots of $A$. porrum and $L$. sativa: Fresh shoot weight of $A$. porrum was the lowest (9.8 $\mathrm{mg}$ ) on MS medium without any source of nitrogen and the highest shoot growth $(32.8 \mathrm{mg}$ ) was obtained on MS medium in which inorganic nitrogen was replaced by casein $(0.1 \%$ and $1 \%$ concentrations) $(P<0.01)$ (Figure $1 \mathrm{~A})$. Growth of $A$. porrum shoot was similar on standard MS medium compared to MS medium without inorganic nitrogen but with $0.01 \%$ casein $(P<0.99)$. Fresh shoot weight of $L$. sativa was the lowest (11 mg) on MS medium without any source of nitrogen and the highest shoot growth $(71.2 \mathrm{mg})$ was obtained on MS medium with inorganic nitrogen (Figure 1 B).

The lowest values of fresh weight of roots of $A$. porrum (2.7 mg) were obtained for seedlings growing on MS without nitrogen; the rest of medium variants gave higher results (from 5 to $6.5 \mathrm{mg}$ ), but similar to each other (no statistical differences) (Figure $1 \mathrm{~A}$ ). The lowest fresh weight of roots of $L$. sativa (2.6 mg) was obtained for seedlings growing on MS without nitrogen, higher values were obtained for seedlings cultivated on standard MS medium and MS with $0.01 \%$ casein (6 and $8.2 \mathrm{mg}$, respectively), but the highest $L$. sativa root fresh weight was obtained for seedlings growing on MS with $0.1 \%$ or $1 \%$ casein (10.5 and $11.2 \mathrm{mg}$, respecitvely) (Figure $1 \mathrm{~B}$ ).

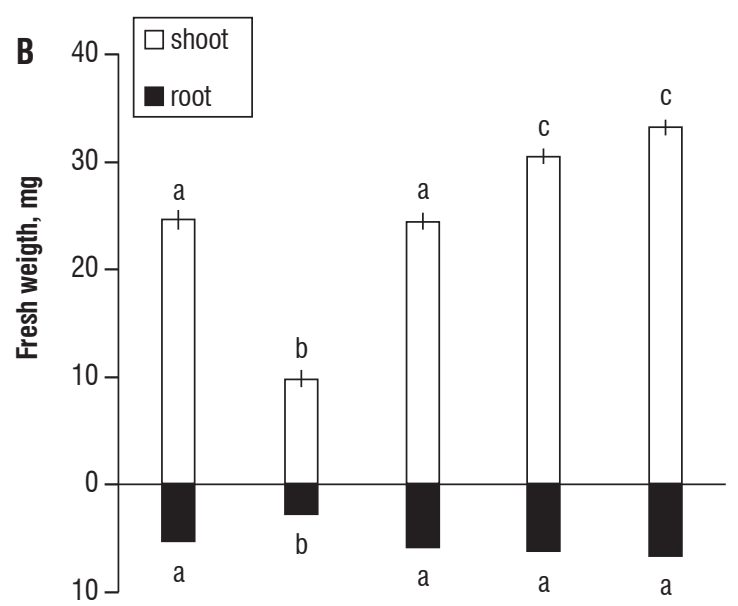

Figure 1. Fresh weight of $L$. sativa (1A) and $A$. porrum seedlings (1B) of seedlings cultivated on different media: (1) Murashige and Skoog medium, (2) MS medium without inorganic nitrogen, (3) MS medium without inorganic nitrogen, but with $0.01 \%$ casein, (4) MS medium without inorganic nitrogen, but with $0.1 \%$ casein, (5) MS medium without inorganic nitrogen, but with $1 \%$ casein. Values are means of six replicates. Error bars indicate standard error of the mean. Statistically significant differences $(P<0.05)$ are indicated by different letters.

\section{Comparison of the level of growth of $A$. porrum with that}

of $L$ sativa: To compare the level of growth of $A$. porrum and L. sativa we presented the results also in the form of percent of growth in comparison with growth on standard MS medium, which was treated as $100 \%$ of growth (Figure 2A). Inter-species comparison of shoot weight revealed that $A$. porrum seedlings obtained significantly higher level of growth on MS media with casein in comparison with the level of growth of shoot of $L$. sativa $(P<0.05)$. In the same way we also presented root growth (Figure 2B). In comparison with root fresh weight of $A$. porrum, $L$. sativa roots obtained higher weight growing on media with casein (statistically significant for media with $0.1 \%$ and $1 \%$ casein). 
A

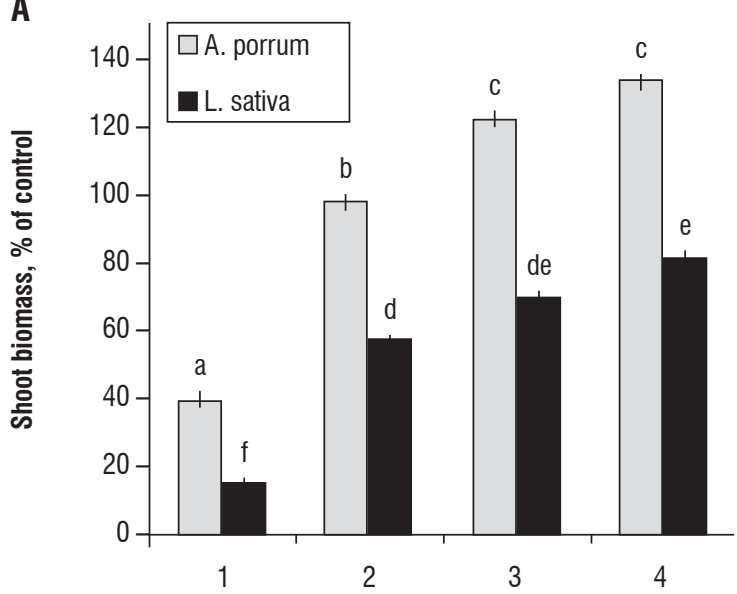

B

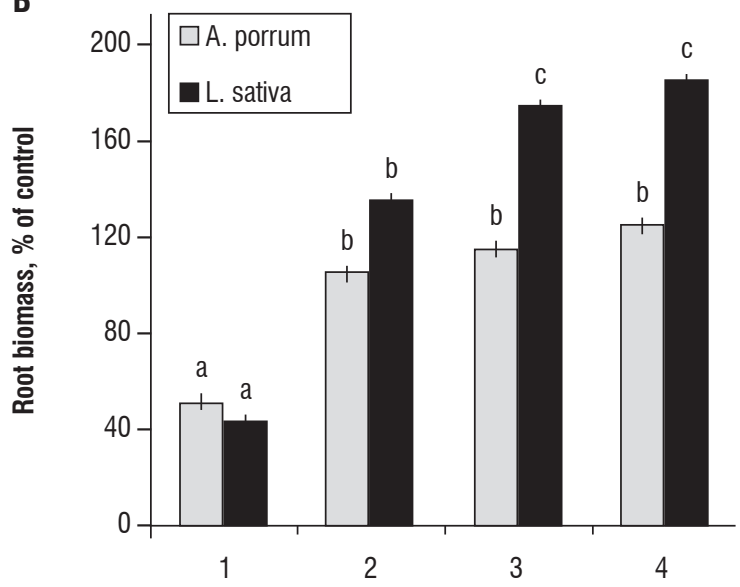

Figure 2. Comparison of growth of Lactuca sativa and Allium porrum shoot (2A) and root (2B) on different culture media: (1) MS medium without inorganic nitrogen, (2) MS medium without inorganic nitrogen, but with $0.01 \%$ casein, (3) MS medium without inorganic nitrogen, but with $0.1 \%$ casein, (4) MS medium without inorganic nitrogen, but with $1 \%$ casein. Results are presented as a percent of growth on standard MS medium (growth on standard MS medium was treated as $100 \%)$. Values are means of six replicates. Error bars indicate standard error of the mean. Statistically significant differences $(P<0.05)$ are indicated by different letters.

Proteolytic activity in the culture medium: Allium porrum seedlings obtained 15 -fold higher proteolytic activity than L. sativa (Figure 3). There were always statistically significant differences between activities obtained for $L$. sativa and $A$. porrum in each culture medium (not shown on figure). Lactuca sativa and $A$. porrum seedlings showed higher proteolytic activities growing on media with casein, especially in higher concentrations (for $1 \%$ casein concentration - 0.006 and $0.124 \mathrm{U} \mathrm{cm}^{2}$ of proteolytic activity, respectively), in comparison with media with inorganic nitrogen (0.002 and $0.026 \mathrm{U} \mathrm{cm}^{2}$ of proteolytic activity, respectively); but in the case of $A$. porrum, that increase in activity was greater.

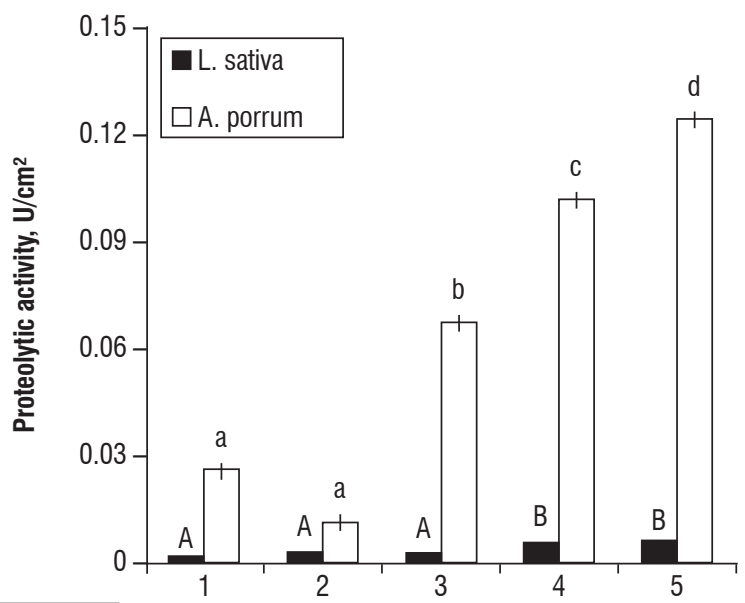

Figure 3. Proteolytic activity in the culture medium of seedlings cultivated on different media: (1) Murashige and Skoog medium, (2) MS medium without inorganic nitrogen, (3) MS medium without inorganic nitrogen, but with $0.01 \%$ casein, (4) MS medium without inorganic nitrogen, but with $0.1 \%$ casein, (5) MS medium without inorganic nitrogen, but with $1 \%$ casein. Values are means of six replicates. Error bars indicate standard error of the mean. Statistically significant differences $(P<0.05)$ between $A$. porrum media are indicated by different letters and significant differences and between $L$. sativa media are indicated by capitals. There were always statistically significant differences between activities obtained for $L$. sativa and $A$. porrum in each culture medium (not shown on figure).

\section{DISCUSSION}

There is increasing interest in developing ecologicalfriendly methods for plant cultivation. A primary means of attaining this goal is to decrease the use of inorganic nitrogen fertilizers, which contribute to environmental pollution (Huang et al., 2003; Wang et al., 2004). Plant assimilation of organic nitrogen (reviewed by Näsholm et al., 2009) provides a means for maintaining high plant yield and reducing environmental pollution. Plant species can differ in the ability to use soil organic nitrogen sources (i.e. Okamoto and Okada, 2004).

According to our earlier paper, plants growing in the same conditions can differ in the level of proteolytic activity in the culture medium, i.e. Allium porrum showed several fold higher proteolytic activity than L. sativa (Godlewski and Adamczyk, 2007). In this paper, we expanded that observation by the use of different variants on MS media (with inorganic or with organic nitrogen source). Both plant 
species (A. porrum and L. sativa) were able to use casein as nitrogen source and in the case of both species addition of protein to the culture medium resulted in significant increase of proteolytic activity. However, A. porrum was significantly more effective at growing on casein as a nitrogen source than $L$. sativa; such result was underlined by comparison of shoot growth of these two species as a percent of growth (growth on standard MS medium was treated as 100\%) (Figure 2A). However, similar comparison of the level of root growth showed different pattern - roots of $L$. sativa obtained higher fresh weight than roots of $A$. porrum. Increased growth of roots in comparison with shoots can point to nitrogen deficiency (Robinson and Rorison, 1988; Ameziane et al., 1995) in L. sativa seedlings cultivated on medium with organic nitrogen.

Plants having higher proteolytic activity in the culture medium should show higher affinity to organic nitrogen sources in the form of amino acids - products of proteolysis. This is in accordance with paper by Matsumoto et al. (1999) in which authors showed that $L$. sativa prefers inorganic over organic nitrogen sources, and with Termine et al. (1987) studies, in which A. porrum plants were growing comparably on inorganic and organic nitrogen sources.

However, one should remember that these studies were conducted in laboratory conditions and in field conditions part of organic soil nitrogen does not exist in an easy accessible form, i.e. in complexes with tannins (Bending and Read, 1996). Moreover, tannins can not only create complexes with soil proteins - substrates for proteolysis, but tannins can also decrease proteolytic activity (He et al., 2006; Adamczyk et al., 2009).

According to our hypotheses, A. porrum seedlings obtained higher shoot fresh weight on medium with casein than $L$. sativa. Significant variations in proteolytic activities in different culture media (highest in $A$. porrum than in $L$. sativa, especially in the case of media with casein) point to mechanism hidden by these differences in plant growth seedlings of $A$. porrum by using higher proteolytic activities in the culture medium than $L$. sativa were strongly increasing the pool of accessible organic nitrogen. Studies conducted on $A$. porrum and $L$. sativa suggest, that also other plant species can effectively use proteins in nitrogen nutrition; however efficiency of this strategy is species-specific.

\section{REFERENCES}

Adamczyk B, Godlewski M, Smolander A, Kitunen V (2009) Degradation of proteins by enzymes exuded by Allium porrum roots - A potentially important strategy for acquiring organic nitrogen by plants. Plant Physiol. Biochem. 47:919-925.

Adamczyk B, Godlewski M, Zimny J, Zimny A (2008) Wheat (Triticum aestivum) seedlings secrete proteases from the roots and, after protein addition, grow well on medium without inorganic nitrogen. Plant Biol. 10:71824.

Adamczyk B, Kitunen V, Smolander A (2009) Polyphenol oxidase, tannase and proteolytic activity in relation to tannin concentration in the soil organic horizon under silver birch and Norway spruce. Soil Biol. Biochem. 41:20852093

Améziane R, Limami MA, Noctor G, Morot-Gaudry J-F (1995) Effect of nitrate concentration during growth on carbon partitioning and sink strength in chicory. J. Exp. Bot. 46:1423-1428.

Bending GD, Read DJ (1996) Nitrogen mobilization from protein-polypheno complex by ericoid and ectomycorrhizal fungi. Soil Biol. Biochem. 28:16031612.

Falkengren-Grerup U, Månsson KF, Olsson MO (2000) Uptake capacity of amino acids by ten grasses and forbs in relation to soil acidity and nitrogen availability. Environ. Exp. Bot. 44:207-219.

Ge T, Song S, Roberts P, Jones DL, Huang D, Iwasaki K (2009) Amino acids as a nitrogen source for tomato seedlings: The use of dual-labeled $\left({ }^{13} \mathrm{C}\right.$, ${ }^{15} \mathrm{~N}$ ) glycine to test for direct uptake by tomato seedlings. Environ. Exp. Bot. 66:357-361.

Godlewski M, Adamczyk B (2007) The ability of plants to secrete proteases by roots. Plant Physiol. Biochem. 45:657-664.

Hano C, Addi M, Fliniaux 0, Bensaddek L, Duverger E, Mesnard F, Lamblin F, Lainea $E$ (2008) Molecular characterization of cell death induced by a compatible interaction between Fusarium oxysporum f. sp. linii and flax (Linum usitatissimum) cells. Plant Physiol. Biochem. 46:590-600.

He Q, Lv Y, Yao K (2006) Effects of tea polyphenols on activities of a-amylase, pepsin, trypsin and lipase. Food Chem. 101:1178-1182.

Head GC (1966) Estimating seasonal changes in the quantity of white unsuberized root on fruit trees. J. Hortic. Sci. 41:197-206.

Hirner A, Ladwig F, Stransky H, Okumoto S, Keinath M, Harms A, Frommer WB, Koch W (2006) Arabidopsis LHT1 is a high-affinity transporter for cellular amino acid uptake in both root epidermis and leaf mesophyll. Plant Cell 18:1931-1946.

Huang XP, Huang LM, Yue WZ (2003) The characteristics of nutrients and eutrophication in the Pearl River estuary, South China. Mar. Poll. Bull. 47:3036.

Jones DL, Shannon D, Junvee-Fortune T, Farrar JF (2005) Plant capture of free amino acids is maximized under high soil amino acid concentrations. Soil Biol. Biochem. 37:179-181.

Kaye JP, Hart SC (1997) Competition for nitrogen between plants and soil microorganisms. Tree 12:139-143.

Lipson D, Näsholm T (2001) The unexpected versatility of plants: organic nitrogen use and availability in terrestrial ecosystems. Oecologia 128:305316.

Matsumoto S, Ae N, Yamagata M (1999) Nitrogen Uptake Response of Vegetable Crops to Organic Materials. Soil Sci. Plant Nutr. 45:269-278.

Murashige T, Skoog $F$ (1962) A revised medium for rapid growth and bioassays with tobacco tissue cultures. Physiol. Plantarum 15:473-497.

Näsholm T, Kielland K, Ganeteg U (2009) Uptake of organic nitrogen by plants. New Phytol. 182:31-48.

Okamoto M, Okada K (2004) Differential responses of growth and nitrogen uptake to organic nitrogen in four gramineous crops. J. Exp. Bot. 55:1577585. 
Paungfoo-Lonhienne C, Lonhienne TGA, Rentsch D, Robinson N, Christie M, Webb RI, Gamage HK, Caroll BJ, Schenk PM, Schmidt S (2008) Plants can use protein as nitrogen source without assistance from other organisms. PNAS 105:4524-4529.

Paungfoo-Lonhienne C, Schenk PM, Lonhienne TGA, Brackin R, Meier S, Rentsch D, Schmidt S (2009) Nitrogen affects cluster root formation and expression of putative peptide transporters. J. Exp. Bot. 60:2665-2676.

Persson J, Näsholm T (2001) Amino acid uptake: a widespread ability among boreal forest plants. Ecol. Lett. 4:434-438.

Rentsch D, Schmidt S, Tegeder M (2007) Transporters for uptake and allocation of organic nitrogen compounds in plants. FEBS Lett. 581:22812289.

Reeve JR, Smith JL, Carpenter-Boggs L, Reganold JP (2008) Soil-based cycling and differential uptake of amino acids by three species of strawberry (Fragaria spp.) plants. Soil Biol. Biochem. 40:2547-2552.

Robinson D, Rorison IH (1988) Plasticity in grass species in relation to nitrogen supply. Funct. Ecol. 2:249-257.

Rojas JA, Cruz C, Mikan JF, Villalba LS, de Garcia MCC, Restrepo S (2009) Isoenzyme characterization of proteases and amylases and partial purification of proteases from filamentous fungi causing biodeterioration of industrial paper. Inter. Biodeter. Biodegr. 63:169-175.

Sautheil L, Glaser B, Weigelt A (2009) Uptake of intact amino acids by plants depends on soil amino acid concentrations. Environ. Exp. Bot. 66:145-152.

Svennerstam H, Ganeteg U, Bellini C, Näsholm T (2007) Comprehensive screening of Arabidopsis mutants suggests the lysine histidine transporter 1 to be involved in plant uptake of amino acids. Plant Physiol. 143:1853-1860.
Termine E, Lairon D, Taupier-Letage B, Gautier S, Lafont R, Lafont H (1987) Yield and content in nitrates minerals and ascorbic acid of leek and turnips grown under mineral or organic fertilizations. Plant Foods Hum. Nutr. 37:321332.

Tomarelli RM, Charney J, Harding ML (1949) The use of azoalbumin as a substrate in the colorimetric determination of peptic and tryptic activity. J. Lab. Clin. Med. 34:428-433.

Tsay Y-F, Chiu C-C, Tsai C-B, Ho C-H, Hsu P-K (2007) Nitrate transporters and peptide transporters. FEBS Lett. 581:2290-2300.

Wang DJ, Liu Q, Lin JH, Sun RJ (2004) Optimum nitrogen use and reduced nitrogen loss of production of rice and wheat in the Yangtse Delta region. Environ. Geochem. Health 26:221-227.

Weigelt A, Bol R, Bardgett RD (2005) Preferential uptake of soil nitrogen forms by grassland plant species. Oecologia 142:627-635.

Weigelt A, King R, Bol R, Bardgett RD (2003) Inter-specific variability in organic nitrogen uptake of three temperate grassland species. J. Plant Nutr. Soil Sci. 166:606-611.

Xu X, Ouyang H, Cao G, Pei Z, Zhou C (2004) Uptake of organic nitrogen by eight dominant plant species in Kobresia meadows. Nutr. Cycl. Agroecosys. 69:5-10.

Zhang X, Zhang L, Ye G, Wang Y, Chen Y, Chen D (2007) The impact of introducing the Bacillus thuringiensis gene into cotton on boll nitrogen metabolism. Environ. Exp. Bot. 61:175-180. 